\title{
Bowman layer transplantation in the treatment of keratoconus
}

\author{
Diana C. Dragnea ${ }^{1,2}$, Rénuka S. Birbal ${ }^{1,2,3}$, Lisanne Ham ${ }^{1,2,3}$, Isabel Dapena ${ }^{1,2}$, Silke Oellerich ${ }^{1}$, Korine van Dijk ${ }^{1,2}$ \\ and Gerrit R. J. Melles ${ }^{1,2,34^{*}}$ (D)
}

\begin{abstract}
Several treatment options corresponding to the grade of keratoconus have been established. These are ultra-violet corneal crosslinking and intracorneal ring segments for mild to moderate keratoconus, and penetrating keratoplasty or deep anterior lamellar keratoplasty for the more advanced cases of keratoconus.

Bowman layer transplantation was developed as a procedure for patients with advanced, progressive keratoconus. The technique consists of transplanting an isolated donor Bowman layer into a mid-stromal pocket of a keratoconic cornea resulting in corneal flattening and stabilization against further ectasia. Thus, it aims at corneal stabilization in eyes with advanced keratoconus, and enabling continued contact lens wear for normal visual functionality. By being a sutureless procedure and using an acellular graft, it potentially avoids commonly known suture and graftrelated complications of penetrating or deep anterior lamellar keratoplasty.

The treatment seems to be a promising option in the management of advanced keratoconus in order to postpone or prevent a more invasive corneal surgery, while minimizing the risk of complications and allowing less stringent surveillance and less intensive medical therapy.
\end{abstract}

Keywords: Cornea, Advanced keratoconus, Bowman layer, Penetrating keratoplasty, Deep anterior lamellar keratoplasty, Crosslinking, Intracorneal ring segments

\section{Background}

Several treatment options corresponding to the grade of keratoconus $(\mathrm{KC})$ have been established [1]. These are ultra-violet corneal crosslinking (UVCXL) and intracorneal ring segments (ICRS) for mild to moderate $\mathrm{KC}$, and penetrating keratoplasty (PK) or deep anterior lamellar keratoplasty (DALK) for the more advanced cases of KC [2-9].

UVCXL has been introduced in 2003 and its purpose is to freeze the evolution of the disease and postpone or prevent the need for corneal transplantation $[2,10,11]$. The results of the procedure have been encouraging, with approximately $80-90 \%$ of treated eyes reaching topographic stability [3], but the procedure may only be indicated in corneas with minimum corneal thickness of $400 \mu \mathrm{m}$. Although techniques are being developed to treat thinner corneas as well [4], it may be less suitable for more advanced $\mathrm{KC}$, given

\footnotetext{
* Correspondence: research@niios.com; http://www.niios.com

${ }^{1}$ Netherlands Institute for Innovative Ocular Surgery (NIIOS), Laan op Zuid 88, 3071AA Rotterdam, The Netherlands

${ }^{2}$ Melles Cornea Clinic, Rotterdam, The Netherlands

Full list of author information is available at the end of the article
}

that the rates of treatment failure and vision-threatening complications may increase [5].

The use of ICRS for KC was first reported in 2000 [6]. By reshaping the cornea, the segments may improve the corneal optics, while it may also confer some amount of support, possibly helping in postponing or avoiding corneal transplantation $[7,12]$. Nevertheless, eyes with severe corneal thinning and steepening may be less eligible for the procedure [8].

For patients with advanced KC, PK and DALK are still currently used as the only options of treatment, in spite of well-known postoperative difficulties such as wound healing and suture related problems, tectonic instability, the risk of allograft rejection, a chronic steroid use that may predispose to cataract formation and glaucoma, and disappointing visual results [1,9]. Advanced $\mathrm{KC}$ patients may, however, still have a subjectively acceptable contact lens (CTL)-corrected vision [13]. Consequently, they may still profit from reshaping the cornea and preventing further $\mathrm{KC}$ progression to enable continued contact lens wear with normal daily visual performance. 
In $\mathrm{KC}$ corneas, changes in the organization of the stromal lamellae and unequal distribution of the collagen fibrillar mass, particularly around the apex of the cone, have been described [14]. Confocal microscopy studies have demonstrated a reduction in the number of keratocytes and stromal lamellae in KC compared to normal subjects, the reduction being greater in more advanced cases of KC [15]. Furthermore, the Bowman layer (BL) of these corneas consistently shows fragmentation, which are then filled with stromal collagen [16].

The physiologic purpose of the BL remains thus far somewhat unclear because numerous eyes have had their BL disrupted by laser refractive procedures without any significant consequences, and congenital absence of the BL has been reported in normal corneas [17]. On the other hand, it has also been suggested that the BL may be the strongest biomechanical element of the human cornea followed by the anterior third of the cornea [18]. As such, the BL may play a structural role in maintaining the shape/ tectonic stability in $\mathrm{KC}$ corneas. Consequently, we hypothesized that a surgical approach in which the possible functionality of the $\mathrm{BL}$ in stabilizing the cornea could be restored, could potentially reinforce these thin and structurally fragile corneas. We developed a surgical technique in which an isolated $\mathrm{BL}$ graft is positioned inside a recipient $\mathrm{KC}$ cornea, sandwiched between the stromal layers above and below, while reshaping the anterior corneal surface in a flatter position [19]. The main treatment objective of the surgery, which was first described in 2014 and is called BL transplantation [19], is halting progression and reducing corneal steepness, which in turn, allows continued daily CTL wear (mainly scleral lenses) and preserve the present CTL-corrected vision, potentially postponing or avoiding the need for PK or DALK [20, 21]. By being a sutureless procedure and using an acellular graft, it potentially avoids all commonly known suture and graft-related complications

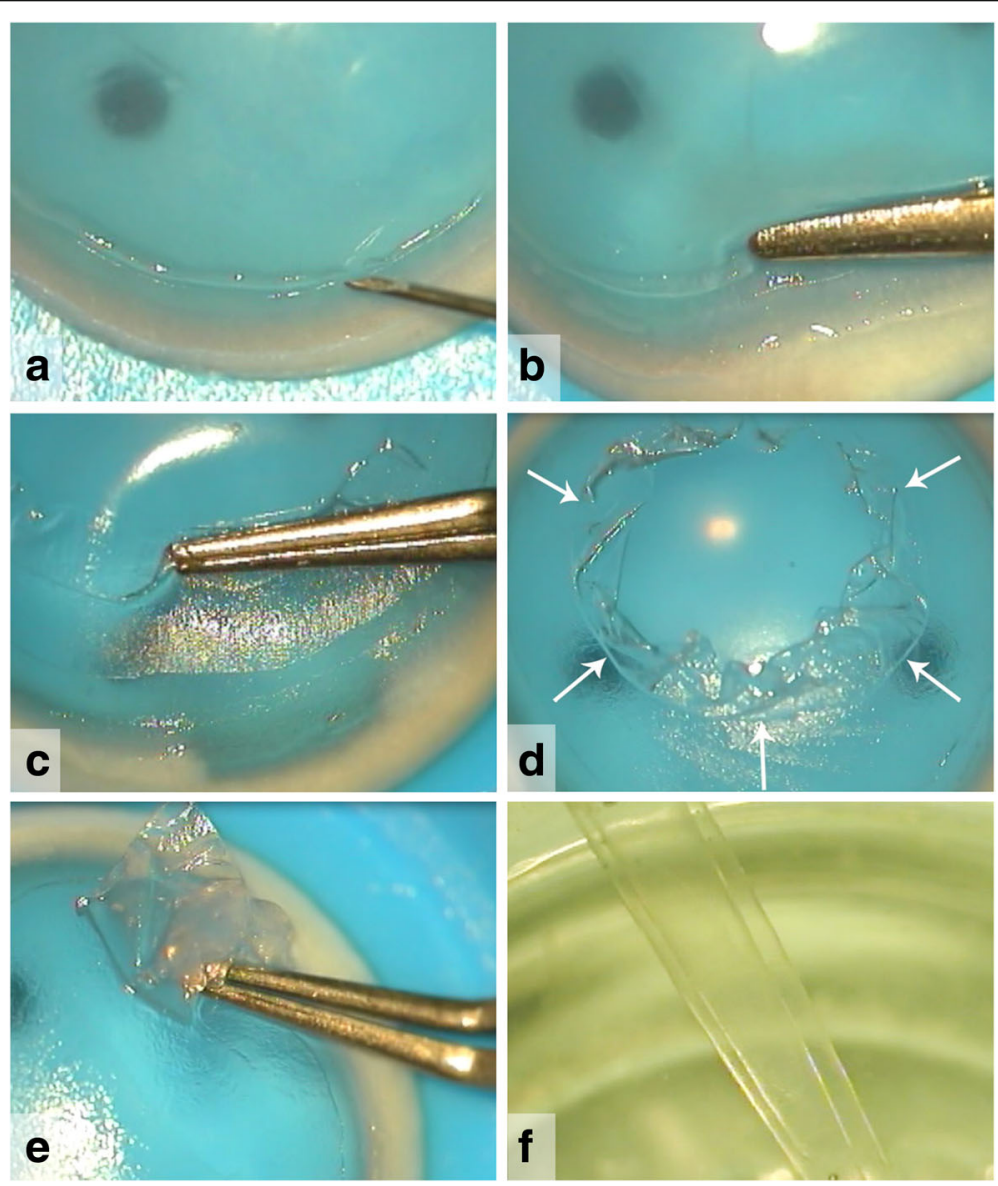

Fig. 1 Surgical views of a Bowman layer (BL) graft preparation. A donor globe is mounted on a globe holder or a donor corneo-scleral rim is placed on an artificial anterior chamber with its epithelial side up. Corneal epithelial cells should be removed, after which, (a) just within the limbal area a superficial incision can be made over $360^{\circ}$ with a $30 \mathrm{G}$ needle. (b) A peripheral donor BL edge is then lifted from the underlying anterior stroma using a single tip of a McPherson forceps. (c-e) Subsequently, by grasping the BL edge with the McPherson forceps via gentle slow movements in a circular manner, the entire BL is carefully peeled away to free the tissue from its underlying attachments. After preparation, the $B L$ graft is evaluated and can be trephined if needed. (f) The BL graft tends roll into a single or double roll due to tissue elasticity 
of earlier techniques such as penetrating or deep anterior lamellar keratoplasty (PK and DALK, respectively) [1].

$\mathrm{BL}$ transplantation is indicated in eyes with progressive advanced keratoconus that are no longer eligible for UVCXL or ICRS given the corneal thickness or steepness. Potential candidates should have "acceptable" CTL-corrected vision and documented KC progression. What represents "acceptable" vision is likely to depend on the patient: in our experience, many patients would rather like to preserve the vision they have than undergo a PK/DALK with its risks and postoperative burden.

\section{Review}

\section{Graft preparation}

BL graft preparation was initially described in 2010 [22], and has remained largely unchanged since: BL grafts can either be prepared from whole donor globes (obtained less than $24 \mathrm{~h}$ postmortem, with corneas considered ineligible for PK) or from an anterior corneal button after stripping the Descemet membrane and endothelium for use in patients who were indicated for Descemet Membrane Endothelial Keratoplasty (DMEK) [23]. Donor globes or anterior corneal buttons (epithelial side up) are mounted on a globe holder or artificial anterior chamber, respectively, and the epithelium is removed using surgical spears (Fig. 1). Then, a 30-gauge needle is used to incise the $\mathrm{BL}$, just within the limbal area, $360^{\circ}$ around. The peripheral BL can be lifted and grasped with a McPherson forceps and then carefully peeled free from the underlying stroma, obtaining a 9- to $11-\mathrm{mm}$ diameter BL graft (Fig. 1) [23]. Due to the inherent elasticity of the tissue, BL grafts tend to curl into a single or double roll with the epithelial border at the outside (Fig. 1). In the end, the donor BL graft is rinsed in 70\% alcohol for $30 \mathrm{~s}$ and stored in organ-culture medium until the time of transplantation [23].

Recently, the use of a femtosecond laser for donor BL preparation has been evaluated. Preliminary results were encouraging, showing that the laser cut tissues demonstrated smoother / more regular edges compared to those prepared manually. However, the femtosecond laser prepared grafts were significantly thicker, containing some amounts of anterior stroma. The potential optical impact of these differences in graft morphology is presently unknown [24].

\section{Surgical technique}

BL transplantations are performed under local anesthesia with the patient positioned in anti-Trendelenburg position, after an ocular massage and a Honan's balloon for $10 \mathrm{~min}$. The first step of the surgery is a superior conjunctival peritomy. Then $1-2 \mathrm{~mm}$ outside the limbus, a $5 \mathrm{~mm}$ partial thickness scleral tunnel is made and dissected up into the clear cornea using a crescent knife. Subsequently, a paracentesis is created and the anterior chamber is filled with air (Fig. 2) [19]. After this step, a manually dissected stromal pocket is created over $360^{\circ}$ up to the limbus, using the technique as described for manual DALK [25]. For BL transplantation, a 50\% dissection depth, which can be found using the "air-endothelial reflex" [25] is preferred (to minimize the risk of unintentional

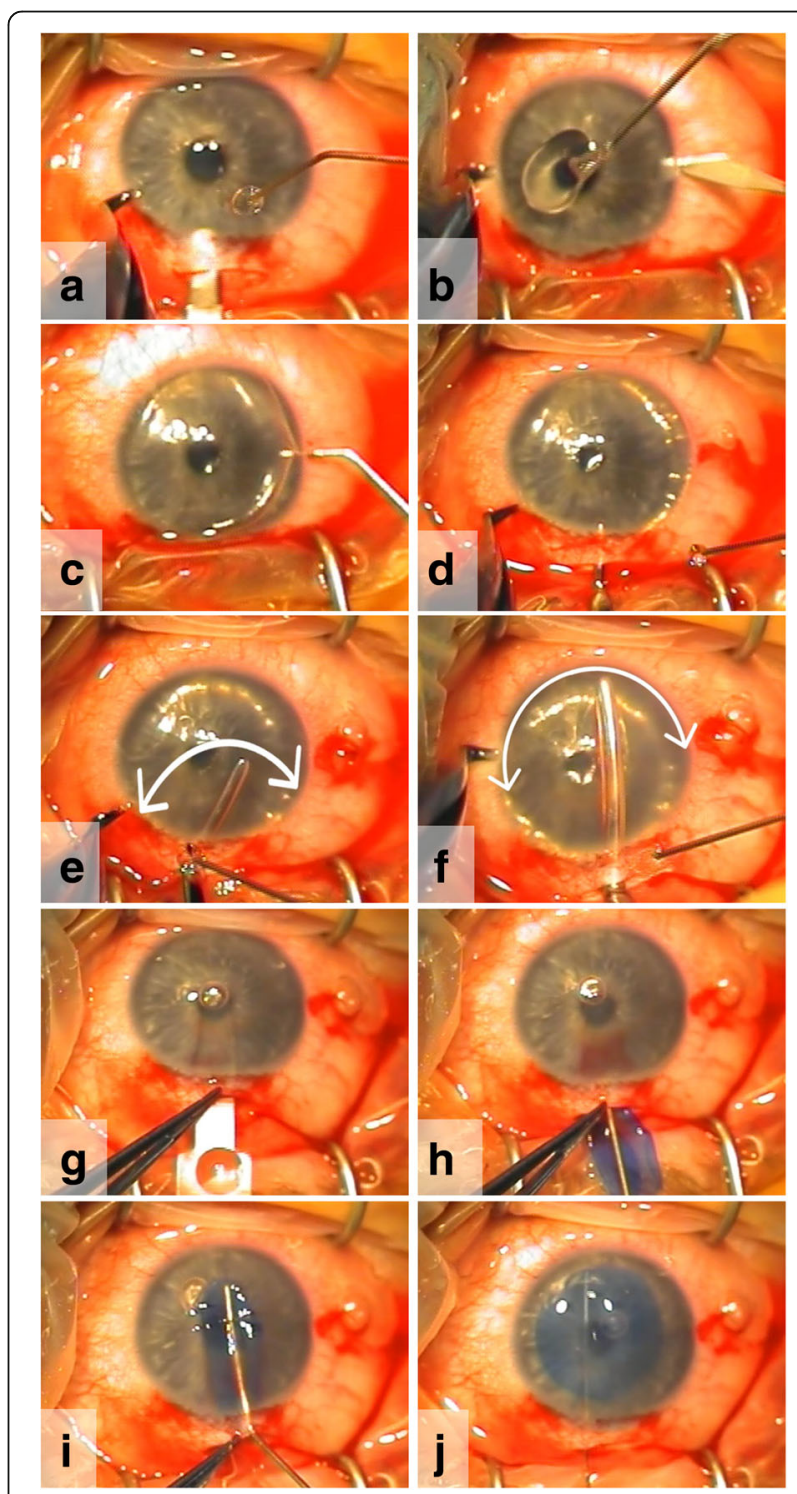

Fig. 2 Intraoperative video-stills of a Bowman layer (BL) transplantation. (a) A scleral tunnel incision and (b) paracenteses are made. (c) Then, the anterior chamber is filled with air, and (d-f) a manually dissected mid-stromal pocket is created, using different sizes spatulas. (f) As an indication for the dissection depth, the 'thin black line' alongside the spatula can be used. After removing most air from the anterior chamber, $(\mathbf{g}, \mathbf{h})$ the BL graft is inserted into the pocket atop of a glide, and (i) then carefully unfolded and centered with an 30G cannula. (j) After complete unfolding and positioning, the BL graft is sandwiched between the anterior and posterior stromal layers. No sutures are necessary to fixate the graft or to close the tunnel incision 
anterior or posterior perforation) (Fig. 2). Once this has been accomplished, most air is removed from the anterior chamber, and a surgical glide is threaded through the corneo-scleral incision into the dissected pocket. At the same time, the BL graft is again immersed in $70 \%$ ethanol for $30 \mathrm{~s}$ to remove all remnant cellular material, after which it is thoroughly rinsed with balanced salt solution (BSS), stained with Trypan blue, and placed atop of the glide, where it is pushed into the eye with the help of a cannula (Fig. 2). Once the graft is inside
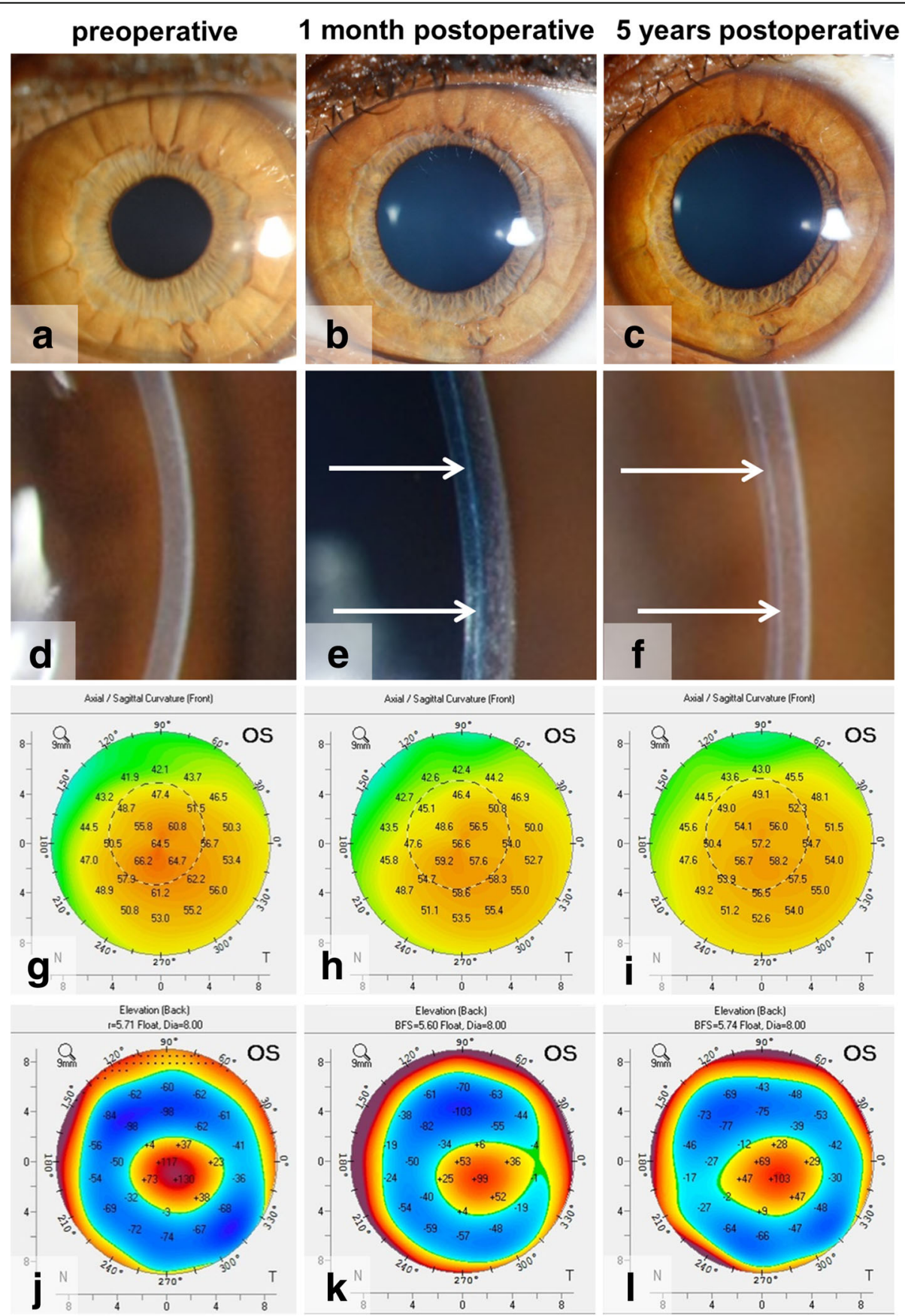

Fig. 3 Pre- and postoperative clinical images of an eye that underwent BL transplantation. (a-f) Slit-lamp pictures, ( $\mathbf{g}-\mathbf{i})$ anterior corneal topography and (j-I) posterior corneal elevation maps of an eye, $(\mathbf{a}, \mathbf{d}, \mathbf{g}, \mathbf{j})$ before and $(\mathbf{b}, \mathbf{e}, \mathbf{h}, \mathbf{k})$ at one month and $(\mathbf{c}, \mathbf{f}, \mathbf{i}, \mathbf{l}) 5$ years after Bowman layer (BL) transplantation. Slit-lamp imaging demonstrates (a) a clear cornea preoperatively, as well as postoperatively at (b) 1 month and $(\mathbf{c}) 5$ years, while $(\mathbf{e}, \mathbf{f})$ the BL graft is visible as a thin white line within the host stroma (white arrows). (g-i) Corneal topography shows a flattening from (g) preoperatively to (h) the 1 month follow-up, and (h, i) stabilization thereafter. Likewise, (j-I) a decrease in posterior corneal elevation can be noticed from $(\mathbf{j}, \mathbf{k})$ before to 1 month postoperatively, after which $(\mathbf{k}, \mathbf{I})$ no changes occur up to 5 years after BL transplantation 
the stromal pocket, the glide is removed, and the graft is unfolded and positioned by manipulating it with the cannula, jets of BSS, and indentation on the corneal surface (Fig. 2). After a complete unfolding and positioning of the graft, the eye is pressurized with BSS, the conjunctiva is repositioned to the superior limbus and the eye is patched. No sutures are required.

Potential difficulties in the learning curve of the surgical technique are the midstromal manual dissection of these thin $\mathrm{KC}$ corneas, together with the graft handling. As the same manual dissection technique is used as with manual DALK, some experience with the manual DALK-surgery could be beneficial for the learning curve in performing a BL transplantation. Furthermore, familiarity with DMEK may aid in BL graft handling.

Postoperative medications include antibiotics for one week and a corticosteroid for the first month, after which the steroid may be tapered according to the surgeon's discretion $[19,20]$.

\section{Clinical outcomes}

BL transplantation is a relatively new KC treatment option with limited literature available. Single center clinical outcomes for BL transplantation are available up to 7 years after the surgery [21,26]. In a first series of eyes in which BL transplantation was performed, operated eyes showed a significant flattening of the cornea of 8-9 $\mathrm{D}$ (on average) in maximum keratometry values in the first postoperative month, after which stabilization of the ectasia has been observed (Fig. 3) [20, 21]. Likewise, the posterior corneal curvature flattens and stabilizes thereafter (Fig. 3) [20]. Meanwhile, on slit-lamp examination the graft remains only vaguely visible as a thin white line (Fig. 3) [19-21].

Within a reported 5-year follow-up period, KC disease progression and/or severe complications could be avoided in $84 \%$ of the eyes [21]. The flattening of the cornea was accompanied by an improvement in spectacle-corrected visual acuity and a decrease in corneal higher order aberrations (especially spherical aberration) [27]. The mid-stromal positioning of the $\mathrm{BL}$ graft may however give some increase in corneal backscatter [27], which was found to occur up to 5 years after BL transplantation [21], and is possibly initiated by interface irregularities and/or differences in refractive indices between the BL graft and host stroma. Nevertheless, the clinical impact of this rise in corneal densitometry may be minimal given the objective and subjective lack of visual disturbance. In fact, CTL-corrected vision showed no changes from before to after BL transplantation [20, 21], and patients sometimes even experienced an improvement in their functional vision, since CTL wear became more comfortable as a result of the large amount of postoperative corneal flattening.

\section{Complications}

So far, our experience with BL transplantation showed that the surgery may be a promising, minimally invasive approach to arrest KC progression; surgical manipulations are limited to the pocket within the recipient corneal stroma, no surface incisions are made, and no sutures are used to fixate the graft. Therefore, unlike PK or DALK, postoperative ocular surface complications or suture related problems do not occur. Furthermore, since the BL tissue is acellular, allograft reaction may be unlikely and topical steroids may be rapidly discontinued, minimizing the risk of glaucoma development or cataract formation.

The main intraoperative complication (the only encountered) may be a Descemet membrane perforation while dissecting the mid-stromal pocket, which is described to occur in 10\% ( 2 out of a first series of 22 eyes) of the originally operated cohort of BL transplantation eyes $[19,20]$. As with DALK, these perforations may be managed expectantly by aborting the operation, to allow healing, and re-attempting again at a later date. Alternatively, the surgeon may proceed with $\mathrm{PK}$, depending on the size and position of the perforation [28].

Postoperatively, an unexpected complication is the occurrence of a corneal hydrops at 4.5, 6 and 6.5 years postoperatively in two patients ( 3 eyes), in a series of 20 eyes after successful BL transplantation. These patients had a history of severe eye rubbing and atopy and developed the hydrops despite no evidence of progressive steepening or thinning [26]. Therefore, also after BL transplantation, patients should be counseled about the possible impact of eye-rubbing, and allergies may need closer monitoring and treatment. No other postoperative complications have been observed [21, 26].

\section{Conclusion}

BL transplantation aims at corneal stabilization in eyes with advanced $\mathrm{KC}$, enabling continued CTL wear for normal visual functionality. The treatment seems like a promising option for the management of advanced $\mathrm{KC}$ in order to postpone or prevent a more invasive corneal surgery, while minimizing the risk of (long-term) complications and allowing less stringent surveillance and less intensive medical therapy.

\section{Abbreviations \\ BL: Bowman layer; BSS: Balanced salt solution; CTL: Contact lens; D: Diopters; DALK: Deep anterior lamellar keratoplasty; DMEK: Descemet membrane endothelial keratoplasty; ICRS: Intracorneal ring segments; KC: Keratoconus; PK: Penetrating keratoplasty; UVCXL: Ultra-violet corneal crosslinking}

Financial disclosure

Dr. Melles is a consultant for DORC International and SurgiCube International. Dr. Dapena is a consultant for DORC International. No other author has a financial or proprietary interest in any material or method mentioned. 


\section{Authors' contributions}

Drafting of the manuscript (DCD, KvD, GRJM), literature search (all authors), preparing Figs. (DCD, KvD, LH), critical revision of the manuscript (all authors), final approval of the manuscript (all authors).

\section{Ethics approval and consent to participate}

Not applicable.

\section{Consent for publication}

Not applicable.

\section{Competing interests}

Dr. Melles is a consultant for DORC International and SurgiCube International. Dr. Dapena is a consultant for DORC International. No other author has a financial or proprietary interest in any material or method mentioned.

\section{Author details}

'Netherlands Institute for Innovative Ocular Surgery (NIIOS), Laan op Zuid 88, 3071AA Rotterdam, The Netherlands. ${ }^{2}$ Melles Cornea Clinic, Rotterdam, The Netherlands. ${ }^{3}$ Amnitrans EyeBank, Rotterdam, The Netherlands. ${ }^{4}$ NIIOS-USA, San Diego, USA.

Received: 30 April 2018 Accepted: 27 August 2018

Published online: 12 September 2018

\section{References}

1. Parker JS, van Dijk K, Melles GR. Treatment options for advanced keratoconus: a review. Surv Ophthalmol. 2015;60:459-80.

2. Wollensak G, Spoerl E, Seiler T. Riboflavin/ultraviolet-a-induced collagen crosslinking for the treatment of keratoconus. Am J Ophthalmol. 2003; 135:620-7.

3. Raiskup-Wolf F, Hoyer A, Spoerl E, Pillunat LE. Collagen crosslinking with riboflavin and ultraviolet-A light in keratoconus: long-term results. J Cataract Refract Surg. 2008:34:796-801.

4. Hafezi F, Mrochen M, Iseli HP, Seiler T. Collagen crosslinking with ultravioletA and hypoosmolar riboflavin solution in thin corneas. J Cataract Refract Surg. 2009;35:621-4

5. Wollensak G, Spoerl E, Wilsch M, Seiler T. Endothelial cell damage after riboflavin- ultraviolet-A treatment in the rabbit. J Cataract Refract Surg. 2003; 29:1786-90.

6. Colin J, Cochener B, Savary G, Malet F. Correcting keratoconus with intracorneal rings. J Cataract Refract Surg. 2000;26:1117-22.

7. Bedi R, Touboul D, Pinsard L, Colin J. Refractive and topographic stability of Intacs in eyes with progressive keratoconus: five-year follow-up. J Refract Surg. 2012;28:392-6.

8. Alió $J \mathrm{~L}$, Shabayek MH, Belda Jl, Correas P, Feijoo ED. Analysis of results related to good and bad outcomes of Intacs implantation for keratoconus correction. J Cataract Refract Surg. 2006;32:756-61.

9. Pramanik S, Musch DC, Sutphin JE, Farjo AA. Extended long-term outcomes of penetrating keratoplasty for keratoconus. Ophthalmology. 2006;113:1633-8.

10. Sandvik GF, Thorsrud A, Råen M, Østern AE, Sæthre M, Drolsum L, et al, Does corneal collagen cross-linking reduce the need for keratoplasties in patients with keratoconus? Cornea. 2015:34:991-5.

11. Godefrooij DA, Gans R, Imhof SM, Wisse RP. Nationwide reduction in the number of corneal transplantations for keratoconus following implementation of cross-linking. Acta Ophthalmol. 2016;94:675-8.

12. Piñero DP, Alio JL. Intracorneal ring segments in ectatic corneal disease - a review. Clin Exp Ophthalmol. 2010;38:154-67.

13. Koppen C, Kreps EO, Anthonissen L, Van Hoey M, Dhubhghaill SN, Vermeulen $\mathrm{L}$. Scleral lenses reduce the need of corneal transplants in severe keratoconus. Am J Ophthalmol. 2018;185:43-7.

14. Meek KM, Tuft SJ, Huang Y, Gill PS, Hayes S, Newton RH, et al. Changes in collagen orientation and distribution in keratoconus corneas. Invest Opthalmol Vis Sci. 2005:46:1948-56.

15. Ku JY, Niederer RL, Patel DV, Sherwin T, McGhee CN. Laser scanning in vivo confocal analysis of keratocyte density in keratoconus. Ophthalmology. 2008;115:845-50.

16. Sherwin T, Brookes NH. Morphological changes in keratoconus: pathology or pathogenesis. Clin Exp Ophthalmol. 2004;32:211-7.
17. Kasner L, Mietz H, Green WR. Agenesis of Bowman's layer. A histopathological study of four cases. Cornea. 1993;12(2):163-70.

18. Marshall J. The 2014 Bowman Lecture-Bowman's and Bruch's: a tale of two membranes during the laser revolution. Eye (Lond). 2015;29(1):46-64.

19. van Dijk K, Parker J, Tong CM, Ham L, Lie JT, Groeneveld-van Beek EA, et al. Midstromal isolated Bowman layer graft for reduction of advanced keratoconus: a technique to postpone penetrating or deep anterior lamellar keratoplasty. JAMA Ophthalmol. 2014;132:495-501.

20. van Dijk K, Liarakos VS, Parker J, Ham L, Lie JT, Groeneveld-van Beek EA et al. Bowman layer transplantation to reduce and stabilize progressive, advanced keratoconus. Ophthalmology. 2015;122:909-17.

21. van Dijk K, Parker JS, Baydoun L, Ilyas A, Dapena I, Groeneveld-van Beek EA, et al. Bowman layer transplantation: 5-year results. Graefes Arch Clin Exp Ophthalmol. 2018;256:1151-8.

22. Lie J, Droutsas K, Ham L, Dapena I, Ververs B, Otten H, et al. Isolated Bowman layer transplantation to manage persistent subepithelial haze after excimer laser surface ablation. J Cataract Refract Surg. 2010;36:1036-41.

23. Groeneveld-van Beek EA, Parker J, Lie JT, Bourgonje V, Ham L, van Dijk K, et al. Donor tissue preparation for Bowman layer transplantation. Cornea. 2016:35:1499-502.

24. Parker J, Huls F, Cooper E, Graves P, Groeneveld-van Beek EA, Lie JT, et al. Technical feasibility of isolated Bowman layer graft preparation by femtosecond laser: a pilot study. Eur J Ophthalmol. 2017;27:675-7.

25. Melles G, Lander F, Rietveld FJ, Remeijer L, Beekhuis WH, Binder PS. A new surgical technique for deep stromal, anterior lamellar keratoplasty. $\mathrm{Br} \mathrm{J}$ Ophthalmol. 1999:83:327-33.

26. Zygoura V, Birbal RS, van Dijk K, Parker JS, Baydoun L, Dapena I, et al. Validity of Bowman layer transplantation for keratoconus: visual performance at 5-7 years. Acta Ophthalmol. 2018; https://doi.org/10.1111/aos.13745.

27. Luceri S, Parker J, Dapena I, Baydoun L, Oellerich S, van Dijk K, et al. Corneal densitometry and higher order aberrations after Bowman layer transplantation: 1-year results. Cornea. 2016;35:959-66.

28. Karimian F, Feizi S. Deep anterior lamellar keratoplasty: indications, surgical techniques and complications. Middle East Afr J Ophthalmol. 2010;17:28-37.

\section{Ready to submit your research? Choose BMC and benefit from:}

- fast, convenient online submission

- thorough peer review by experienced researchers in your field

- rapid publication on acceptance

- support for research data, including large and complex data types

- gold Open Access which fosters wider collaboration and increased citations

- maximum visibility for your research: over $100 \mathrm{M}$ website views per year

At BMC, research is always in progress.

Learn more biomedcentral.com/submissions 